\title{
The propagation and backscattering of soliton-like pulses in a chain of quartz beads and related problems. (I). Propagation
}

\author{
Marian Manciu ${ }^{\mathrm{a}, 1}$, Surajit Sen ${ }^{\mathrm{a}, *}$, Alan J. Hurd \\ -Department of Physics, and Center for Advanced Photonic and Electronic Materials, \\ State University of New York at Buffalo, Buffalo, New York, NY 14260-1500, USA \\ 'Department 1841, Sandia National Laboratories, Albuquerque, NM 87185, USA
}

Received 28 June 1999

\begin{abstract}
We confirm that for vanishingly small loading and large impact condition, it may be possible to generate solitons in a chain of grains that are characterized by Hertzian contacts. For uniform or progressive loading conditions throughout the chain, one generates soft-solitons which are weakly dispersive in space and time. Under conditions of weak impact, one generates acoustic pulses through the chain. We describe the displacements, velocities and accelerations suffered by the individual grains when subjected to solitons, soft-solitons and acoustic pulses and describe the effects of restitution on the propagating pulse. (c) 1999 Elsevier Science B.V. All rights reserved.
\end{abstract}

PACS: $46.10 .+z ; 03.40 . \mathrm{Kf} ; 43.25 .+\mathrm{y}$

\section{Introduction}

Solitons are among the most fascinating objects known to the scientist $[1,2]$. From a physical perspective, one can describe the dynamical soliton as a "tight bundle" of energy that can travel through a medium without any dispersion in space or in time. Solitons are encountered in a handful of physical systems and usually, under special initial conditions. As shown first theoretically, simulationally and experimentally by Nesterenko [3-5], granular systems belong to the select category of systems that support

\footnotetext{
* Corresponding author. Fax: +1-716 6452507.

${ }^{1}$ On leave of absence from INFIM, Bucharest-Magurele, Romania.

0378-4371/99/S - see front matter (C) 1999 Elsevier Science B.V. All rights reserved.

PII: S0378-4371(99)00371-4
} 


\section{DISCLAIMER}

This report was prepared as an account of work sponsored by an agency of the United States Government. Neither the United States Government nor any agency thereof, nor any of their employees, make any warranty, express or implied, or assumes any legal liability or responsibility for the accuracy, completeness, or usefulness of any information, apparatus, product, or process disclosed, or represents that its use would not infringe privately owned rights. Reference herein to any specific commercial product, process, or service by trade name, trademark, manufacturer, or otherwise does not necessarily constitute or imply its endorsement, recommendation, or favoring by the United States Government or any agency thereof. The views and opinions of authors expressed herein do not necessarily state or reflect those of the United States Government or any agency thereof. 


\section{DISCLAIMER}

Portions of this document may be illegible in electronic image products. Images are produced from the best available original document. 
solitons. Somehow, the discovery of solitons in granular, or more specifically, Hertzian systems received limited attention during the decade that followed Nesterenko's initial work [3-5]. Recently, Sinkovits and Sen [6,7] reported studies on impulse propagation in granular media in one and two spatial dimensions. Later, Coste et al. [8] reported experiments that confirmed the theoretical and experimental work of Nesterenko [3-5] and was consistent with the studies of Sinkovits and Sen [6,7]. More recently, MacKay [9] has published a proof (as opposed to a solution to the dynamics problem) that Hertzian chains support solitons and Sen and Manciu have proposed a simple formula to describe the grain displacements during the passage of a soliton [10].

The key property of a "Hertzian system" [11] is that two elastic grains in intimate contact would exert mutual repulsion that would be proportional to their overlap raised to some power. Thus, if $2 R$ denotes the diameter of a spherical grain, then the overlap $\delta \geqslant 0$ would be $\delta \equiv 2 R-r_{i, i+1}$, where $r_{i, i+1}$ is the actual distance between the centers of the adjacent grains. The potential energy gained by the compressed two-grain system turns out to be $V(\delta)=a \delta^{n}$ where $a$ is some constant [11]. In Hertzian systems, one can calculate $a$ which depends upon the elastic properties of the media that the grains are constituted of and $n$ which is sensitive to the contact geometry of the grains [12]. Typically, Hertzian systems are anharmonic, i.e., $n>2$ and hence, they exhibit nonlinear dynamics. One expects interesting dynamical behavior in these systems. As we shall see, the problem of acoustic transport through granular systems reveals a variety of interesting phenomena. Under special conditions, one can support solitons in these systems as has been claimed by Nesterenko and others.

A few years ago, Sinkovits and Sen [6,7] carried out particle-dynamics-based analyses of the propagation of sound and shock impulses through one and two-dimensional granular systems that are subjected to gravity. The key objective of these analyses was to confirm that particle-dynamics-based studies could readily recover the predictions regarding acoustic propagation in Hertzian systems subjected to gravity which are based upon elasticity theory [13] and probe the possible non-linear behavior at the shallow reaches of the bed. Among the results presented in Refs. [6,7] was a finding that the kinetic energy of the granular bed, which has been subjected to an impulse at its surface, when plotted as a function of time and space, appeared to travel down the bed as a pulse that suffered very little dispersion. In summary, the gravitationally loaded granular beds that were probed, supported soliton-like excitations when they were subjected to appropriate impulses. A soliton-like object can be envisioned as a softer bundle of energy than a true soliton. It travels like a soliton but experiences some interaction with the medium as it travels. The softness of the energy bundle, or the dispersion suffered by the pulse, is significant even for pulses with relatively small amplitudes.

The presence of soliton-like pulses in perturbed Hertzian systems presents interesting possibilities. One such possibility is to exploit these pulses, which can travel through a granular medium with little dispersion, to probe for buried objects which may be indetectable by electromagnetic, ultrasonic and purely acoustic probes $[14,15]$. Metal-poor landmines, unexploded ordnance, and buried structures in granular beds are examples of 
underground objects that may be detectable using backscattering of soliton-like pulses. We shall discuss our studies on the backscattering of soliton-like pulses from buried objects in the following article [16].

We discuss the following problems in this article: (i) the conditions under which solitons can form; (ii) the conditions under which soliton-like pulses can be sustained and (iii) the conditions under which acoustic pulses can form.

Details of our models and of the particle dynamics simulations are presented in Section 2. The results of our study are presented in Section 3. We close in Section 4 with a summary of the work and open questions.

\section{The model and the simulations}

\subsection{Intergrain interactions}

We model a granular chain as a collection of spheres that are in contact with neighbors via external loading or by gravitational compaction. We follow the classic work of Hertz [11] and describe the energy associated with the repulsive interaction between any two compressed spheres labeled $i$ and $i+1$ of radii $R_{i}$ and $R_{i+1}$ (while they are uncompressed) as follows. We define the "overlap" between the two adjacent grains by $\delta_{i, i+1} \equiv R_{i}+R_{i+1}-r_{i, i+1}$, where we let $r_{i, i+1}$ represent the distance between the centers of the two spheres. The interaction energy between the granular spheres is expressed as a function of this overlap as follows:

$$
V\left(\delta_{i, i+1}\right)=\frac{2}{5 D} \sqrt{\frac{R_{i} R_{i+1}}{R_{i}+R_{i+1}}} \delta_{i, i+1}^{5 / 2} \equiv a \delta_{i, i+1}^{5 / 2},
$$

where the constant $a \equiv 2 / 5 D \sqrt{\left(R_{i} R_{i+1}\right) /\left(R_{i}+R_{i+1}\right)}$ and where

$$
D=\frac{3}{4}\left(\frac{1-\sigma_{i}^{2}}{E_{i}}+\frac{1-\sigma_{i+1}^{2}}{E_{i+1}}\right) \text {, }
$$

in which $\sigma_{i}, \sigma_{i+1}$ and $E_{i}, E_{i+1}$ are the Poisson's ratios and the Young's moduli of the two bodies, respectively. Anharmonicity or deviation from Hookean form in the potential energy in Eq. (1) arises due to purely geometrical effects and can be evaluated for perfectly spherical grains (for a detailed derivation of Eq. (1), see Ref. [11]). As noted by Coste et al. [8], the interaction energy in Eq. (1) is valid when the repulsive force and $\delta_{i, i+1}$ vary slowly in time relative to the time taken by sound to travel through a spherical grain. In other words, we neglect plastic deformation of the spheres in this study. The experimental work of Coste et al. [8] suggests that this is a reasonable approximation for materials such as steel spheres. However, plastic deformation may play an important role in studying sound propagation in softer granular materials. ${ }^{2}$ These effects are not addressed here.

${ }^{2}$ It turns out that for certain materials, the soliton pulse attenuates rapidly, possibly due to large restitution of the material. We are grateful to Prof. V. Nesterenko for discussions regarding this issue. 


\subsection{Equations of motion and initial conditions}

In this article, we focus on the propagation of a perturbation created by an impact on the first grain of a granular chain. We present results in which, (i) the granular chain is uniformly loaded and gravitational compaction is absent, i.e., when the chains are horizontal, and (ii) in which the chain is gravitationally loaded, i.e., the chain is vertical and the load increases with depth. In case (i), loading the grains such that they are in intimate contact with one another is an important issue. The equation of motion of a grain of mass $m$ at location $z_{i}$ for case (i) is given by

$$
\begin{aligned}
& m \ddot{z}_{i}=n a\left[\left\{\Delta_{0}-\left(z_{i}-z_{i-1}\right)\right\}^{n-1}-\left\{\Delta_{0}-\left(z_{i+1}-z_{i}\right)\right\}^{n-1}\right] \quad \text { case (i), } \\
& m \ddot{z}_{i}=n a\left[\left\{\Delta_{0}-\left(z_{i}-z_{i-1}\right)\right\}^{n-1}-\left\{\Delta_{0}-\left(z_{i+1}-z_{i}\right)\right\}^{n-1}\right]-m g \text { case (ii). }
\end{aligned}
$$

where $n=\frac{5}{2}$ for the case of spherical grains in contact. In Eq. (3), we define $\Delta_{0} \equiv$ $R_{i}+R_{i+1}-l_{0}=2 R-l_{0}$ in a homogeneous system and where $l_{0}$ enters due to loading for the horizontal chain problem and we replace $R_{i}+R_{i+1}$ in the definition of $\delta_{i, i+1}$ given above Eq. (1) by $\Delta_{0}$. As we shall see, $l_{0}$ plays an important role in distinguishing between the shock-like perturbations and the softer compression pulses that can be generated down a column with suitable initial impact velocities on the first grain in the chain. For the vertical column problem in case (ii), in which gravitational compaction plays an important role, we add $-m g$ to the right-hand side of Eq. (3).

In case (i), given $\Delta_{0}$, the initial positions of all the grains in the chain are specified. The velocity of the first grain is specified at time $t=0$. The velocities of all the other grains are initially set to zero. For a given loading or $l_{0}$, the velocity of the first grain therefore defines the physical properties of the soliton-like pulse. We discuss these properties in detail in Section 3.

The initial structure of the vertically oriented granular column (case (ii)) is different $[6,7]$ than the horizontal chain. In this case, the grain at the bottom of the chain is most compressed while the one at top is least compressed. Since granular systems, being macroscopic, are largely insensitive to changes in temperature, it is important to determine the ground state of a gravitationally compacted granular column for studying collective phenomena in these systems. We accomplish this as follows. The location of the bottom grain is first fixed and the positions of the remaining grains are set such that the repulsive forces due to the overlap between the adjacent grains exactly equal the forces required to support the grain column. For a system of $N^{\prime}$ grains in which the bottom grain is labelled 1 , the overlap between grains $i$ and $i+1$ is determined as follows [6,7]:

$$
g \sum_{j=i+1}^{N^{\prime}} m_{j}=\frac{5}{2} a \delta_{i, i+1}^{3 / 2} .
$$

The reader may observe that if the gravitationally compacted chain is loaded, then it is essential to take into account the additional repulsive force term that must arise from 
that loading (as described in case (ii) of Eq. (3)) on the left-hand side of Eq. (4) above. We do not consider loaded gravitationally compacted chains in this study.

The results presented in Section 3 below, address the solutions to Eq. (3) with and without gravitational compaction. In the absence of gravitational compaction, Eq. (3) can be analyzed in the strongly nonlinear limit. Typically, such a limit means that the characteristic size of the perturbation is such that the end grains are compressed at least $10^{-3} 2 R$. In this limit, the traveling soliton pulse is symmetric in position, velocity and acceleration about the geometric center of the soliton. For weaker perturbations, as mentioned in Section 1 above, the pulse is asymmetric and becomes dispersive. Such pulses can no longer be thought of as pure solitons but rather as soliton like.

One can extend the $1 D$ chain of Hertzian grains to that of an ordered $3 D$ lattice. In the general case, when grains have different sizes, shapes, densities and positional disorder, the only way to accurately solve the dynamics of the system of coupled non-linear equations is by numerical means. However, if we assume that each layer is completely rigid in the $x-y$ plane, then the equation for each grain can be replaced by a $1 D$ equation for an entire 2D layer of thickness equalling one grain diameter. In this limit, we recover the 1D equation of motion (Eq. (3)) where the $m$ needs to be replaced by the mass of the entire layer. Thus, for a stack of rigid $2 \mathrm{D}$ planes with each plane densely packed, one can argue that it must be possible to propagate solitons along the $c$-axis provided the initial impulse is imparted to the entire surface layer. In the general case, when granular motion is possible along all three directions, one would expect that for sufficiently strong impulses along the $c$-axis one might be able to propagate weakly dispersive soliton-like perturbations. Indeed, as we shall see [16], it is possible to send soliton-like pulses in close-packed 3D granular beds. The dispersion suffered by an impulse possesses rich structure. Backscattering from buried inclusions in such beds can be used to infer information about the buried object [16].

\subsection{Numerical constants}

Our results are based upon numerical integration of the coupled equations of motion for granular chains with $N$ spheres, where $N \sim 10^{3}$. We have used the third-order Gear predictor-corrector algorithm [17] for time integrating the coupled equations of motion of the $N$ grain system.

We chose constant diameter $2 R=10^{-3} \mathrm{~m}$ and constant mass $m=1.41 \times 10^{-6} \mathrm{~kg}$ for the spheres. For the material coefficients, we have used Young's Modulus $E=7.87 \times$ $10^{10} \mathrm{~N} \mathrm{~m}^{-2}$ for quartz, $E=2.0 \times 10^{11} \mathrm{~N} \mathrm{~m}^{-2}$ for steel and $E=1.0 \times 10^{10} \mathrm{~N} \mathrm{~m}^{-2}$ for plastic, and Poisson's ratios of $\sigma=0.144$ for quartz, $\sigma=0.300$ for steel and $\sigma=0.120$ for plastic. With these data, the constant $a$ in the Hertz potential (see Eq. (1)) is $3.39 \times 10^{8} \mathrm{~N} \mathrm{~m}^{-3 / 2}$ for quartz-quartz interaction, $4.96 \times 10^{8} \mathrm{~N} \mathrm{~m}^{-3 / 2}$ for quartz-steel interaction and is $7.60 \times 10^{7} \mathrm{~N} \mathrm{~m}^{-3 / 2}$ for quartz-plastic interaction.

The velocity of propagation of a typical shock wave that will travel through the granular chain which has been initiated by compressing the surface grain by about $10^{-3}$ of its diameter is dependent upon the precise details of the initial impulse. For 
very weak perturbations (i.e., purely acoustic pulses) the velocity of propagation of the perturbation from one grain to another is typically $5 \mathrm{~m} / \mathrm{s}$. In an earlier study [18], we have found that typically, $c \sim v_{\text {imp }}^{0.2}$. These velocities are much less than the sound velocity in quartz (about $6000 \mathrm{~m} / \mathrm{s}$ ). Thus, for our purposes, it is appropriate to neglect the internal distortions of the quartz beads and to treat them as particles.

\section{Solitons and soliton-like pulses in Hertzian Chains}

\subsection{Perfect solitons in the shock regime: $g=0, l_{0}=0$ case}

- We first discuss the problem of propagation of an impulse in a chain of monodisperse Hertzian spheres when there is no loading of the grains, i.e., when $l_{0}=0$ (see Eq. (3)). To simplify matters, we consider a horizontal chain with no gravity. Our numerical solution to Eq. (3) reveals that when an impulse is imparted to a grain at one end of the chain, i.e., at a surface grain, it propagates down the chain at some fixed velocity, the magnitude of which depends upon the amplitude of the initial impulse, a clear signature of non-linear behavior in the shock regime. Let us insist that the surface grain moves at a fixed velocity $v_{\text {imp }}$ at time $t=0$ while all the other grains are held fixed. Typically, we choose $v_{\text {imp }}$ between $10^{-3}$ and $10 \mathrm{~m} / \mathrm{s}$, which are reasonable impulse limits that can be generated by strikers [3-5]. In what follows, we shall mostly refrain from using $v_{\text {imp }}$ to characterize the impulse and use a new quantity, $\eta / l_{0}$, where $\eta$ denotes the maximum displacement of the surface grain with regard to its equilibrium position $z_{N} \equiv r_{N}+\eta$, where $r_{N}$ denotes the equilibrium position of the surface grain at time $t=0, N$ being the total number of grains. To avoid confusion in describing the calculations below, we now redefine $N$ such that the surface grain is numbered grain number 1 (unless stated otherwise). Thus, we consider the propagation of perfect solitons which are realized in the limit $\eta / l_{0} \rightarrow \infty$.

By numerically integrating the coupled equations of motion for the grains in the chain (see Eq. (3)), we probe the propagation of the impulse through the chain as functions of space and time. The propagating impulse typically spans a few grains at a given instant of time and hence possesses a length scale that cannot be accurately probed in the long-wavelength approximation, the regime in which most soliton problems are typically analyzed. Let us temporarily postpone the discussion of the number of grains in the propagating packet of energy and focus our attention on the motion of a typical particle in the chain as the impulse propagates through it.

Fig. 1(a) presents plots of the displacements of two typical grains in the chain measured in $\mu \mathrm{m}$ as a function of time measured in ms. We present results for the 100 th particle of the chain (solid line), which remains in a position of zero displacement from the equilibrium position until the arrival of the impulse and then shifts its position continuously to some maximum displacement of magnitude that is determined by the nature of the impulse. Unless mentioned otherwise, the numbers are not normalized in this study and are shown with regard to the model for quartz grains. The origin of 


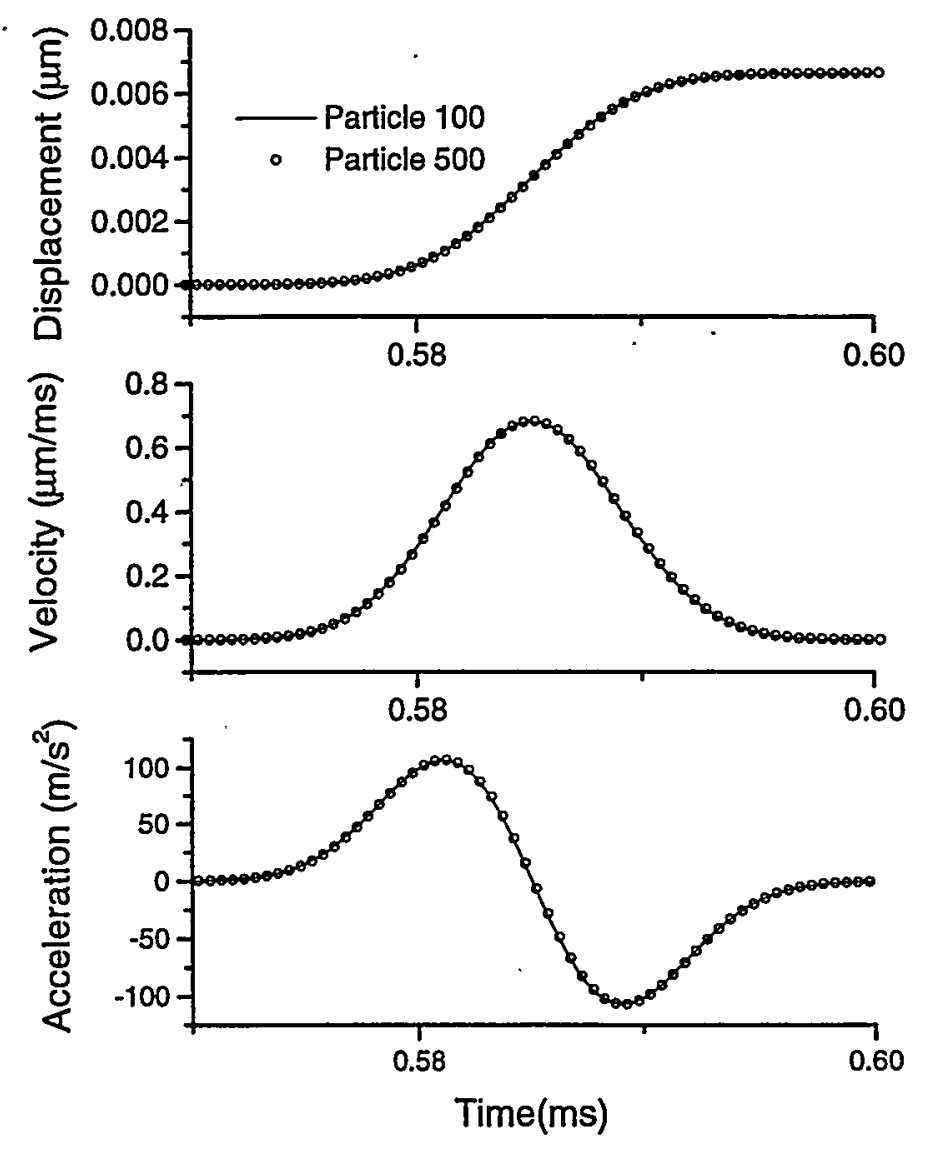

Fig. I. Displacement (upper panel), velocity (middle panel) and acceleration (lower panel) as functions of time for particle numbers 100 (solid line) and 500 (open circles). The dynamical variables for particle 500 are translated in time for the purposes of easy comparison with those of particle 100 and to show that the functional forms of displacement, velocity and acceleration remain invariant for both the particles.

time in Figs. 1(a)-(c) is defined at the starting point of the impulse propagation in particle 1 . The behavior of the displacement function for particle 500 (open circles) turns out to be identical to that of particle 100 , within the limits of our numerical accuracy, when the displacement function for particle 500 is shifted in time so as to coincide with the data for particle 100 . The corresponding data for the velocity and acceleration are shown in Figs. 1(b) and (c).

The data show that there are no measurable differences between the particle displacements, positions and accelerations as functions of time and space and hence we confirm that our calculations reveal that one can propagate solitons through a chain of Hertzian grains when $g=0$ and the chain is not loaded. These findings confirm Nesterenko's results [3-5] and agree with the experimental studies of Coste et al. [8]. We note that prior to this study, the time-dependent behavior of the position, velocity 


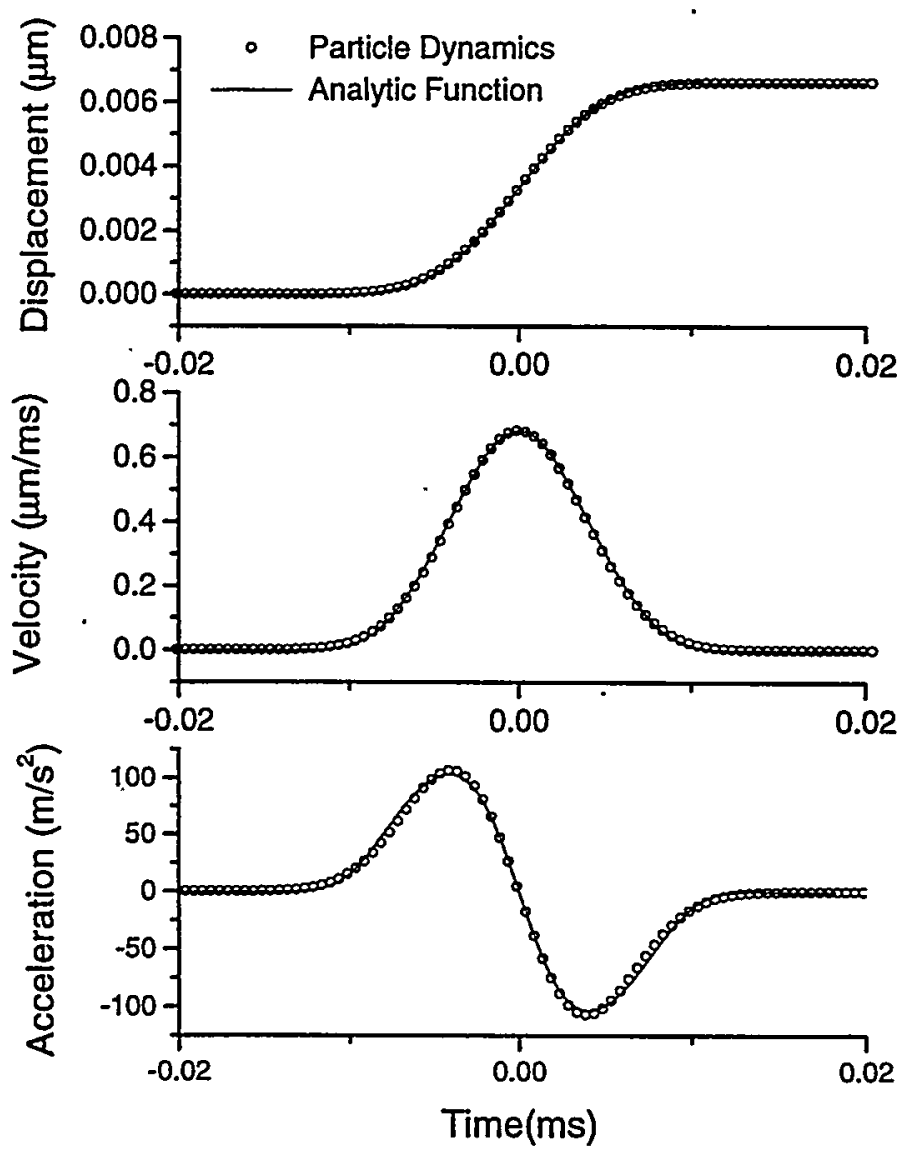

Fig. 2. Displacement (upper panel), velocity (middle panel) and acceleration (lower panel) as functions of time for particle number 100 (open circles) and our analytic result (solid lines). See the text for remarks concerning the time axis. Observe that the agreement between numerical data and analytic formula is worse compared to the agreement between data in Fig. 1 for progressively higher derivatives of the position variable.

and acceleration of the solitons had not been reported in detail in the literature. This is because in the long-wavelength approximation, it is difficult to generate expressions for all of these quantities. As we shall argue below, the knowledge of the behavior of displacements, positions and accelerations of the grains allows us to construct a simple solution to Eq. (3) in the soliton regime [10].

The solid lines in Figs. 2(a), (b) and (c) describe the displacement, velocity and acceleration, respectively, of any grain as a function of time as generated using an approximate analytic solution to the soliton propagation problem. The solution assumes that $z=c t$ in Eq. (3), which allows one to write Eq. (3) for $g=0$ as

$$
m c^{2} \frac{d^{2} \phi_{n}(z)}{\mathrm{d} z^{2}}=n a\left[\left\{\phi_{n}(z-d)-\phi_{n}(z)\right\}^{n-1}-\left\{\phi_{n}(z)-\phi_{n}(z+d)\right\}^{n-1}\right],
$$


where $\phi_{n}(z)$ is the soliton displacement function for any value of $n$ (in these systems, $n>2$ ). The approximate solution can be written as

$$
\phi_{n}(z)=-\frac{A}{2} \tanh \left(\frac{f_{n}(z)}{2}\right),
$$

where $f_{n}(z)=\sum_{q=0}^{\infty} C_{2 q+1}(n) z^{2 q+1}$ and $A$ is determined by the magnitude of the displacement suffered by any grain as a soliton passes through that grain. The details of this solution can be found in Ref. [10].

The agreement between our solution and simulated data (shown in circles in Figs. 2(a)-(c)) is impressive. The reader should observe that the plots are shown using a different origin in time than in Figs. 1(a)-(c). The origin of time used in Figs. 2(a)-(c) is intentionally chosen to be at the center of the soliton to illustrate the symmetry of the soliton in $z$ and hence also in $t$. The derivation of the analytic formula for grain position as a function of space $z$ and hence $t$ is summarized in Ref. [10].

We have also probed the behavior of $\phi_{n}(z)$ for $n=2.2$ and 5. The results are shown in Fig. 3. In Fig. 3, the diamonds $(n=2.2)$, crosses $(n=2.5)$ and squares $(n=5.0)$ display numerically calculated data while the continuous lines are generated using our solution. The values of $C_{2 q+1}$ are generated using the prescription given in Ref. [8]. It becomes progressively difficult to generate analytic solutions as $n$ increases. Our preliminary studies suggest that the slope of the displacement function between -1 and 1 in Fig. 3 increases as $n$ increases. The regions of inflection near -1 and 1 become discontinuous as $n \rightarrow \infty$.

The width $L$ of a soliton (note that the soliton is non-dispersive and hence $L$ is a constant) can be defined by the number of grains over which the energy associated with a propagating soliton resides at a given instant of time. Mathematically speaking, $L$ equals twice the distance between the origin and any of the maxima in Figs. 1(c) or 2(c). It turns out that $L \rightarrow \infty$ when $n \rightarrow 2$ (see Fig. 4). In this limit, one no longer obtains a soliton solution to Eq. (3). This is an expected result in view of the fact that $n=2$ leads to a harmonic equation of motion (see Eq. (3)). When $n \rightarrow \infty$, i.e., when the repulsive interaction between grains in contact approaches the hard core limit, $L \rightarrow 1$ (see Fig. 4). In this limit, one obtains the smallest possible soliton. Typically, however, $n$ varies between 2.5 and 3 and hence the soliton width is about five grains. Hertzian solitons are hence of finite extent and it is therefore difficult to describe the solitons in Hertzian systems using long wavelength analysis. Such analysis was first carried out by Nesterenko [3-5]. Nesterenko managed to obtain a rather good description of the soliton width even within the continuum approximation. In Fig. 4(a), we present numerical data on the width of the soliton (diamonds) and present the width as predicted by Nesterenko's formula given by

$$
L=\frac{R \pi}{(n-2)} \sqrt{n(n-1) / 6},
$$

using the dashed line. The agreement between the simulated data and the numerical results grow worse for $n>3$ or so. Thus, the long wavelength analysis based calculations are not highly reliable as the repulsive potential becomes dominant. Fig. 4(b) 


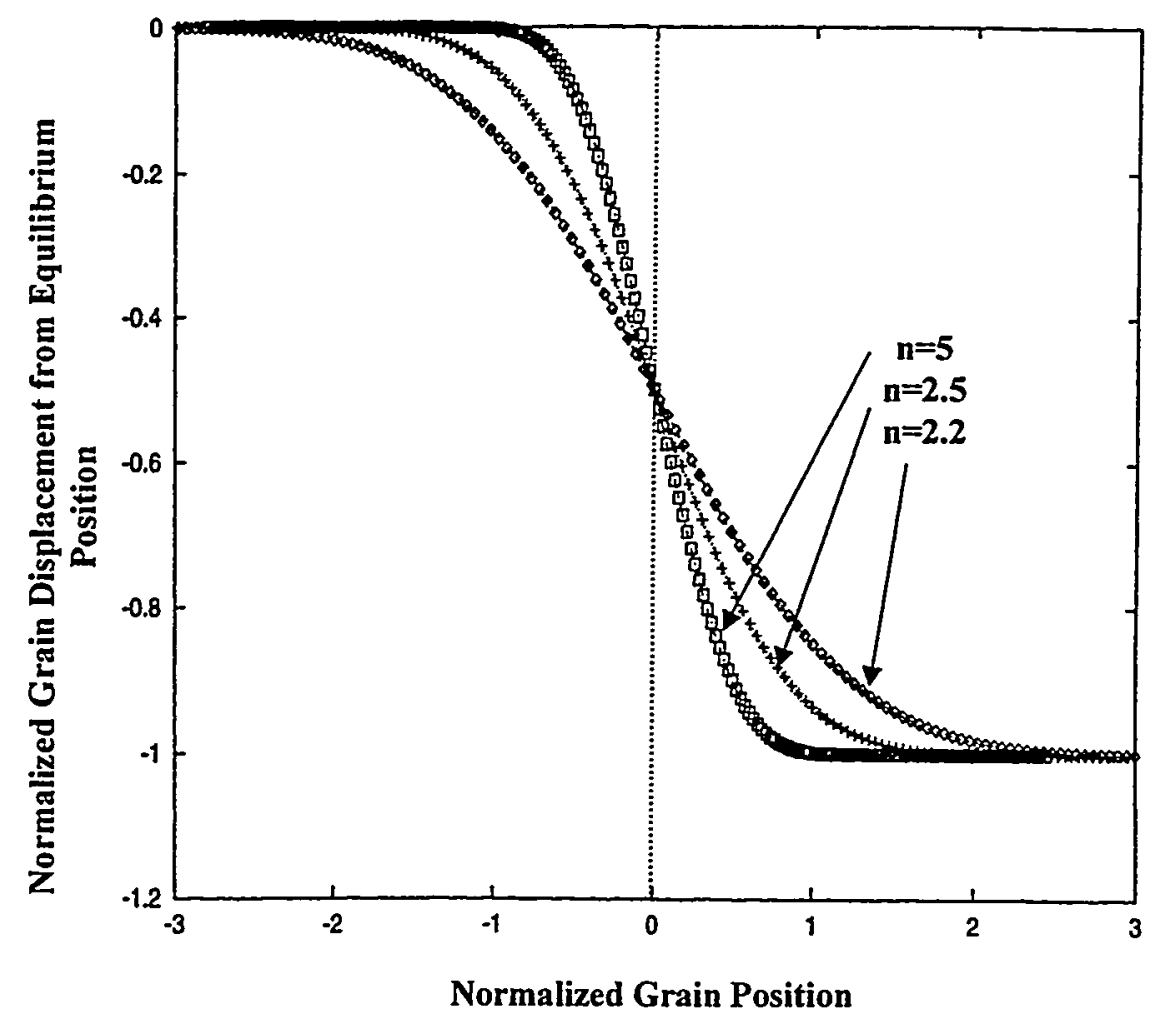

Fig. 3. Normalized displacement function of grains plotted to display the symmetric nature of the displacement function for various values of $n$. Th continuous lines are plots using our analytic formula using various $C_{2 q+1}$ 's.

presents the same study on a $\log -\log$ scale in which the $L-1$ axis has been shown across 8 decades and the $n-2$ axis has been shown across 4 decades. A possible functional form that could fit the data across such a broad spectrum is $1 / \sqrt{\sinh [5(n-2)]}$ (dashed line). Nesterenko's formula yields a straight line on such a log-log scale and is therefore too simplistic for the large $n$ regime.

It is well known in the soliton literature [1] that two distinct solitons that are initiated from opposite directions in a continuous medium pass through each other without any interaction. It is therefore important to study the problem of two crossing solitons initiated from opposite ends of a Hertzian chain. We describe a preliminary study of the interaction of two solitons with unequal velocities that are generated at the opposite ends of a chain and which intersect one another as they simultaneously propagate towards the respective opposite ends of the chain. The calculations showing the velocities of the grains as functions of time and space are depicted in Fig. 5. The sign of velocity depends upon the direction of propagation of the impulse in the Hertzian chain problem. Because compression of grains is required to obtain the solitons, one 

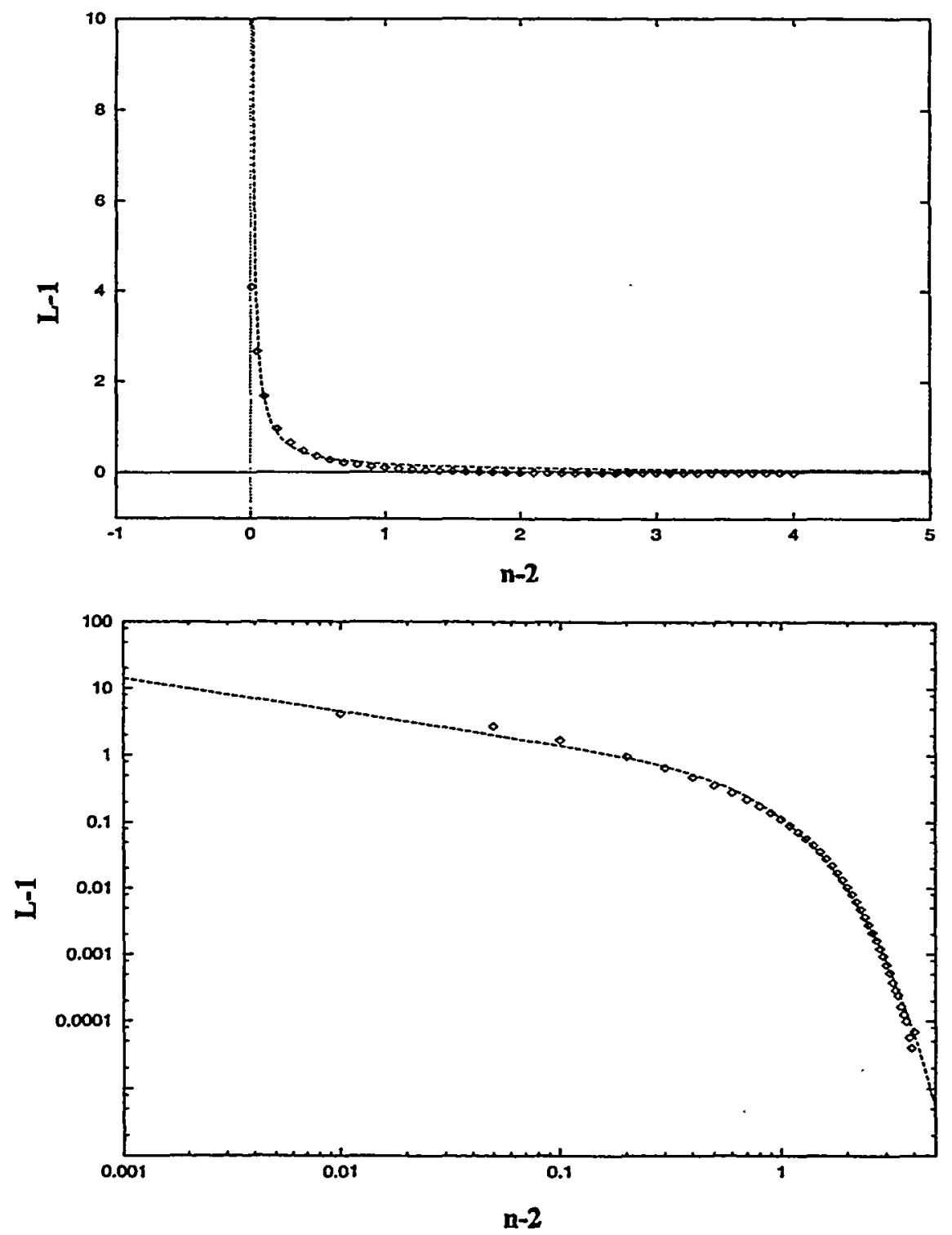

Fig. 4. (Upper panel) Plot of $L-1, L$ being the width of the solitons, for various values of $n$. The plot shows that no solitons can be sustained as $n \rightarrow 2$, the harmonic limit. The dashed line is obtained using Nesterenko's formula (Eq. (7)). (Lower panel) same plot as in the upper panel but displayed in log-log scale. The fitted line is discussed in the text.

cannot create a dilatational pulse for Hertzian chains. Thus, the signs of the velocity variable must always be in opposition for two solitons which are propagating in opposite directions. The vertical axis represents the velocity of the grains in arbitrary units. The planar axes are, grain depth, expressed in terms of grain diameters, and time. 


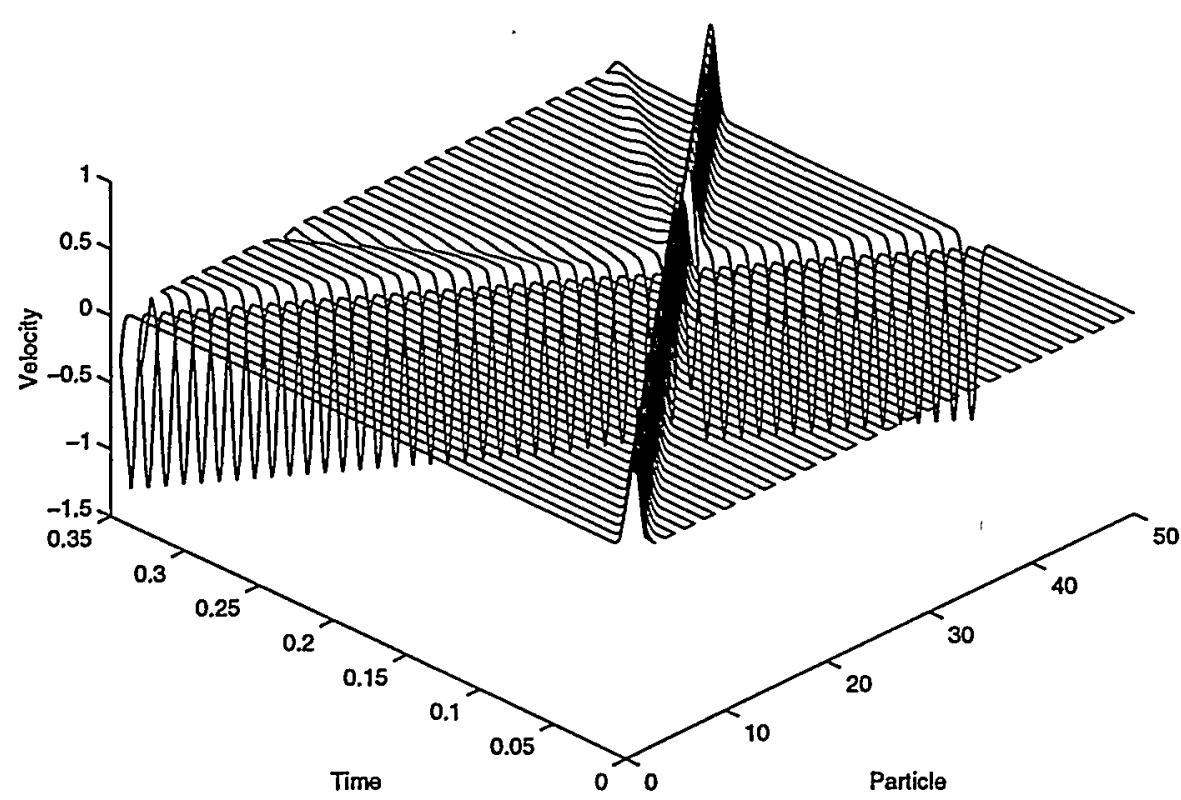

Fig. 5. Two solitons of different sizes are simultaneousiy released from the two ends of a column. The crossing of solitons exhibit peculiar behavior in a discrete medium. In this case, secondary solitons are spawned at the intersection point due to extended "rattling" of the grains where the solitons meet. This kind of behavior is peculiar to the discrete nature of the medium. The units are arbitrary along the time and velocity axes. The particle axis denotes particle position in terms of the particle diameter.

The data shown in Fig. 5 and in Figs. 6(a) and (b) reveal a surprising new result. The solitons interact very weakly as they intersect one another and spawn new secondary solitons, which are much weaker in magnitude and hence move at much slower velocities. Fig. 6(a) shows the kinetic energies of the grains as functions of time as the two opposing solitons intersect. With low enough resolution in the energy scale, the secondary solitons are invisible and it appears as though the motion of the grain where the collision occurs is simply the difference of the two velocities. Fig. 6(b) presents the same data but with much higher resolution in energy. Three distinct secondary solitons emanating from the point of intersection are visible. We are unable to find a simple scaling law for the soliton sizes for the general problem of collision of unequal solitons. Our preliminary analyses show that the energy contained in the secondary solitons that are emitted from the point of intersection is typically less than $0.5 \%$ of the energy contained in the original solitons.

We conjecture that this small energy fraction will become vanishingly small if, somehow, the size of the solitons can be increased; i.e., we expect to recover non-interacting intersections of the solitons for large enough soliton sizes. Increasing the soliton size is only possible in our problem as one approaches the $n \rightarrow 2$ limit. Detailed analysis to examine this conjecture is needed. Such calculations must be carried out with high precision over very large time windows to observe the slowest moving secondary 


\section{Crossing of Solitons from Opposite Ends}

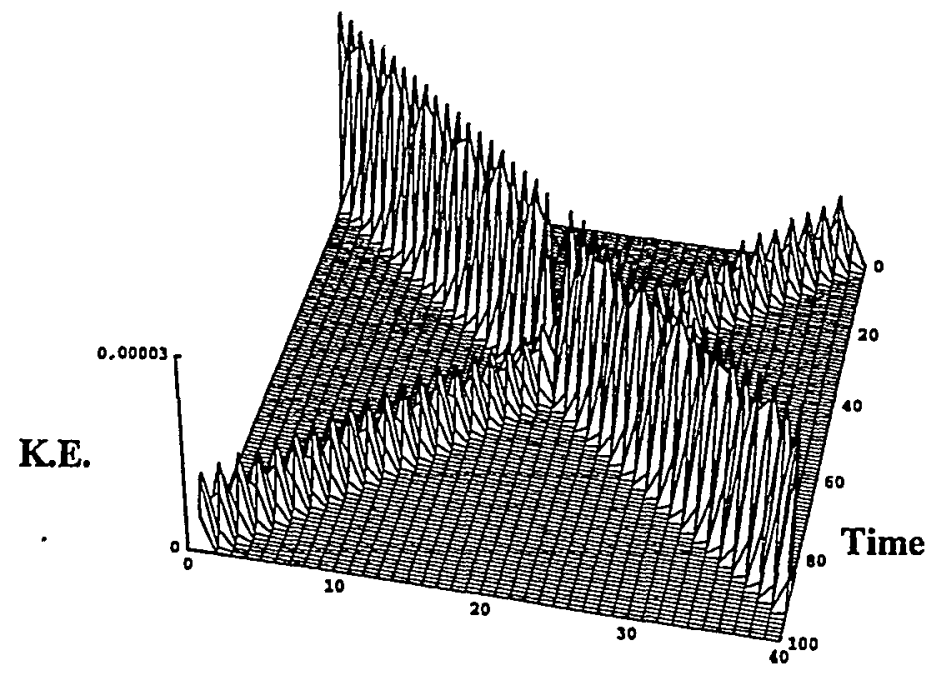

(a)

$$
\text { Depth in Grain Diameters }
$$

\section{Crossing of Solitons from Opposite Ends}

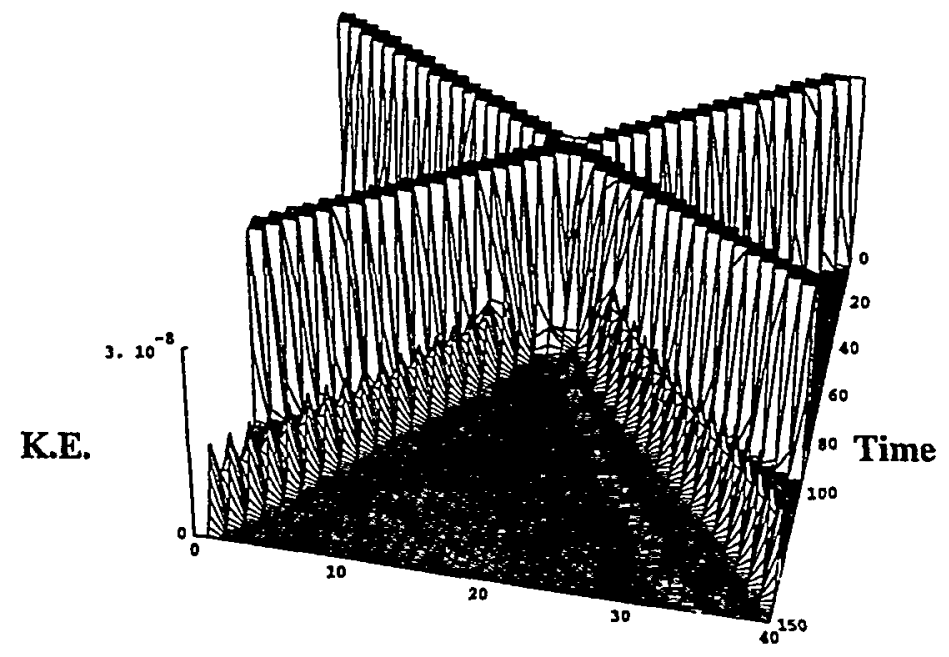

(b)

\section{Depth in Grain Diameters}

Fig. 6. (a) Crossing of unequal solitons initiated at opposite ends of the chain. The kinetic energy of each grain is plotted as functions of space and time. (b) Same data as in (a) but plotted with much higher resolution. The data are shown in program units (see text). 


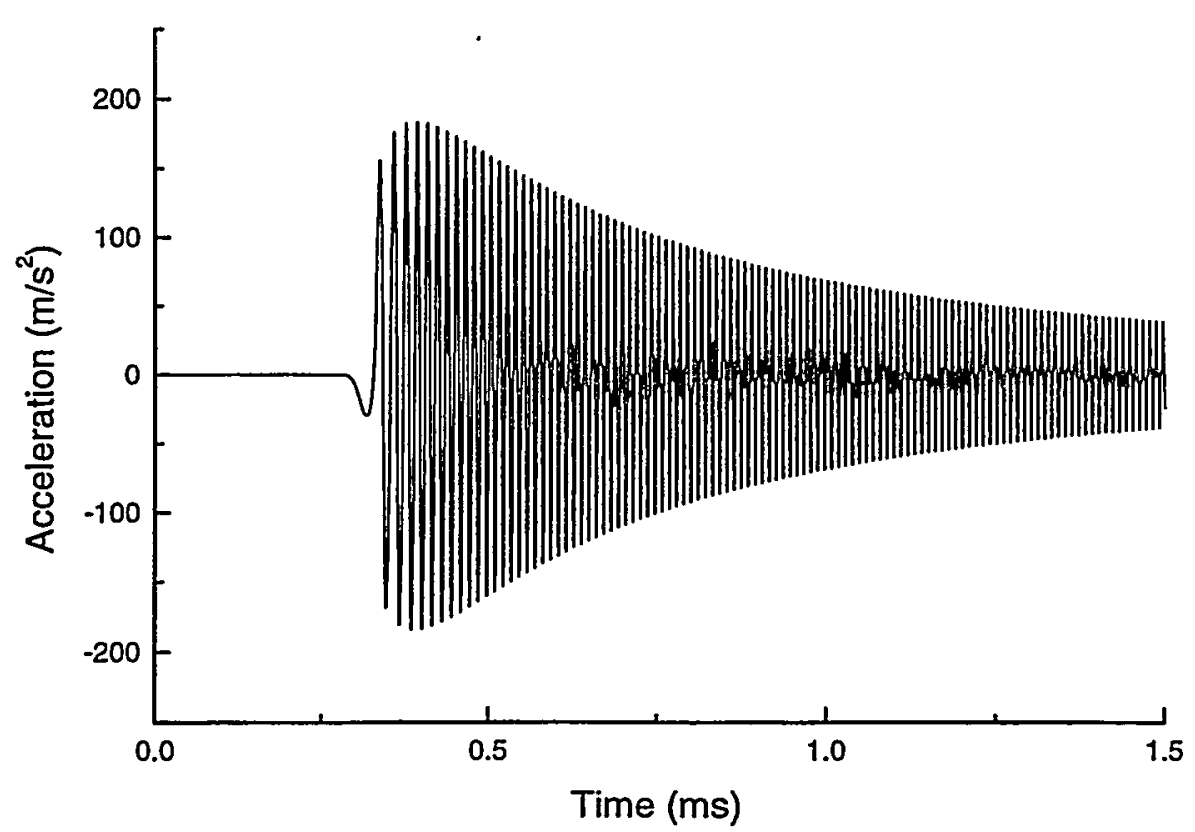

Fig. 7. Acceleration versus time in a loaded (but horizontal) chain for the 100th grain from the surface and very small constant loading (see text). The tail of the velocity envelope, as described by our data, decays as $t^{-1.4}$.

solitons with the smallest velocities and are therefore challenging. Studies are in progress and will be reported in a separate dedicated publication [19].

\subsection{Soliton-like objects: $g=0, l_{0}>0$ and at $g>0$}

As one compresses the grains very gently against one another in a Hertzian chain at $g=0$, one introduces a finite $l_{0}$ into Eq. (3). In our calculations, $l_{0}$ is $10^{-2} \mu \mathrm{m}$. We also choose $\eta=10^{-4} \mu \mathrm{m}$ to be much less than $l_{0}$ and hence consider impulse propagation in the acoustic regime. The consequence of such loading is that the shape of the soliton-like pulse thus formed, which passes through any chosen grain as a function of time, is no longer of the symmetric forms presented in Figs. 1 and 2. Instead, in loaded Hertzian chains, the acceleration suffered by any grain as a function of time, consists of a pulse similar to the one in Fig. 1(c) followed by a long decaying tail in time as shown in Fig. 7. The data are taken for particle number 100 from the surface and a very small $\eta=10^{-4} \mu \mathrm{m}$ has been used. The nature of the decay of the tail can be described by two distinct power laws. Our data reveal that the amplitude of the maximum velocity of the pulse decays with $z$ as

$$
A_{\max }=0.129 z^{-0.5} \text {. }
$$


while the envelope of the pulse experienced by a single grain decays in time as $t$, for large $t$ as

$$
L t_{t \rightarrow \infty}|v| \sim t^{-1.4}
$$

The tail is measured in such a way that the behavior of the leading edge of the pulse does not affect the measurement of decay of the tail. The tail elongates in time as the pulse travels in a scale invariant fashion. We should mention here that it is significantly more challenging to construct an analytic description of the velocity or acceleration of an individual grain in a granular chain compared to the task of describing the characteristics of the propagating energy bundle [20].

In the following discussions, we first describe the nature of the acceleration function for cases with progressive loading (due to gravitation) for various initial impact conditions, followed by a description of the nature of the acceleration autocorrelation function for the various cases of loading and initial impact. The acceleration autocorrelation function, defined below as $C(t)$, for a tagged particle can be used to measure the relaxation of that tagged particle [21-24] as it is perturbed by an impulse and as it tries to return to its unperturbed lower energy state. It serves as a measure of the memory of the interaction suffered by the tagged particle.

The dynamical behavior of the grains is of much interest when $g>0$ and the grains experience progressively larger loadings as a function of depth due to gravitational compression (see Eq. (4)). This is what happens in a vertical Hertzian chain in which gravitational loading plays an important role. In Fig. 8, we plot the accelerations of the 100 th and the 300th grains as functions of time for the case with terrestrial $g$ (assumed to be $9.8 \mathrm{~m} / \mathrm{s}^{2}$ ). It is evident from the data in Fig. 8 (a)-(c) that with decreasing $\eta$, the amplitude of the soliton-like pulse (given in $\mathrm{m} / \mathrm{s}^{2}$ ) attenuates progressively slowly in time and the tail of the pulse becomes more oscillatory in time. In Fig. 8(a), we set $\eta=1 \mu \mathrm{m}$ (strong perturbation), and at $\eta=10^{-2}$ and $10^{-4} \mu \mathrm{m}$ for the cases in Figs. 8(b) and (c). The behavior seen in Fig. 8(c) is obtained for smaller values of $\eta$ as well.

It is evident that the soliton-like solution obtained by solving Eq. (3) with $g>0$ possesses characteristics of a non-dispersive soliton as well as that of a dispersive acoustic wave. For $g=0, l_{0}=0$ one obtains a soliton, while for $g=0, l_{0}>0$ and for $g>0, l_{0}=0$, one obtains soliton-like pulses that are "soft" bundles of energy that disperse slowly in time and in space due to interactions with the medium. The nature of dispersion is strongly dependent upon $\eta$ for both cases until one considers compressions that are less than $10^{-4}$ or so of the grain diameters.

A convenient way of probing the return of each grain to an equilibrium state (which is often not the original equilibrium state) is to calculate the acceleration autocorrelation function. In granular systems, the thermal energies are negligible compared to the potential energies of interest and, hence, it is only meaningful to probe relaxation as a quantity at zero thermal energies. We set acceleration $a(t)$ of some chosen grain, which is sufficiently far from the chain ends, to be carefully probed as a dynamical variable of interest. We then define the normalized relaxation function for the acceleration and 


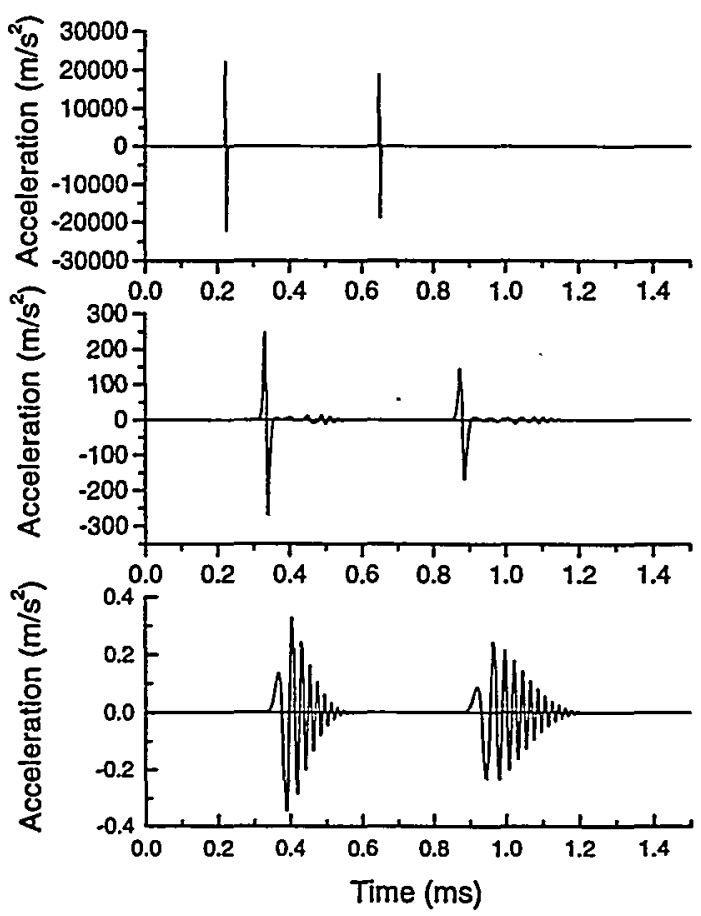

Fig. 8. The figures show acceleration versus time behavior for particles 100 and 350 along a vertical chain for different initial impact conditions. Upper panel deseribes the case with $\eta=1 \mu \mathrm{m}$ (strong perturbation), central panel describes the case with $\eta=10^{-2} \mu \mathrm{m}$ and the lower panel describes the case with $\eta=10^{-4} \mu \mathrm{m}$. Observe the evolution of the tail structure that is characteristics of gravitationally loaded systems as $\eta$ decreases.

write as follows:

$$
C(t) \equiv a(t) a(0) / a(0)^{2} .
$$

The autocorrelation function is calculated by computing

$$
(1 / T) \int_{0}^{T} a\left(t^{\prime}\right) a\left(t^{\prime}+t\right) \mathrm{d} t^{\prime} /(1 / T) \int_{0}^{T} a\left(t^{\prime}\right) a\left(t^{\prime}\right) \mathrm{d} t^{\prime},
$$

for a large number of $t$ values where $T$ is the total time measured from the instant at which the pulse reaches the chosen grain to the maximum time up to which the calculations can been performed without picking up any reflection signals from the chain end. In all instances, the time windows probed are long enough for studying return to equilibrium.

If $C(t) \rightarrow 0$ at short times and if the system returns to the original equilibrium state then one can conclude that the grain has lost all information about the perturbation or the pulse that passed through it. In all of our calculations shown in Fig. 9 we find that $C(t) \rightarrow 0$. This is an expected result in view of the fact that for our non-dissipative, monodisperse system, we do not expect any energy to get trapped and hence ergodicity 


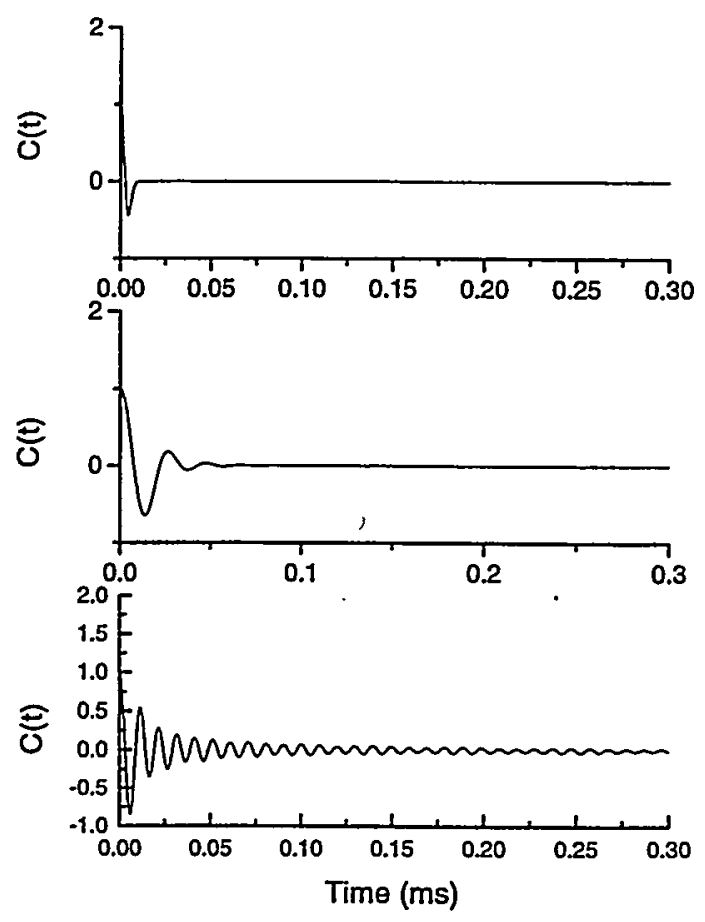

Fig. 9. Acceleration autocorrelation functions (which measure the "memory" of the grain of getting perturbed) versus time(s) for particle 100 (from the surface) in a vertical chain. Upper panel describes on-site autocorrelation for $\eta=1 \mu \mathrm{m}$ (large perturbation and hence soliton-like pulse propagation), central panel describes the case with $\eta=10^{-4} \mu \mathrm{m}$ and the bottom panel describes relaxation when the perturbation is infinitesimally weak such that the particle returns to the equilibrium position (see text).

demands that $C(t) \rightarrow 0$ at $t \rightarrow \infty$ [25]. However, it is important to observe that the tagged grain does not necessarily return to the original equilibrium state unless the perturbation imparted to the grain being studied is infinitesimally weak.

We have probed $C(t)$ for a typical grain, again the 100th grain from the surface, for cases with $g=0, l_{0}=0$ and $\eta=1 \mu \mathrm{m}$ in Fig. 9(a), for $g=9.8 \mathrm{~m} / \mathrm{s}^{2}$ with $\eta=$ $10^{-4} \mu \mathrm{m}$ in Fig. 9(b) (i.e., progressive loading case, the reader may refer to the data in Fig. 8(c) in studying the behavior shown here) and for $\eta / l_{0}=1$ with $l_{0}=10^{-2} \mu \mathrm{m}$ and $g=0$ in Fig. 9 (c) (i.e., constant loading case). In Figs. 9(a) and (b), the tagged grain does not return to the original equilibrium state within the period of calculation even though $C(t) \rightarrow 0$. The distance between the adjacent grains remain altered in these cases long after the pulse has passed through the system. Figs. 9(a) and (b) describe the system memory in the non-linear acoustic regime where the memory of the original equilibrium state is lost. We are presently not aware of a simple way to characterize multi-point correlation functions in the non-linear acoustic regime. To our knowledge, the concepts of linear response theory clearly need revision in describing relaxation processes such as those in Figs. 9(a) and (b) [26,27]. 
In Fig. $9(\mathrm{c})$, however, the tagged grain asymptotically returns to the original equilibrium state in the limit when the perturbation is infinitesimally weak. Thus, Fig. 9(c) describes the system in the linear acoustic regime.

\section{Summary and conclusion}

The present article extends the research reported in Ref. [18] as listed. (1) We present our work on soliton propagation in chains of Hertzian grains for arbitrary $n$ and propose an approximate solution to the grain displacement function as functions of time and space as a soliton passes through the grain. (2) We present data on the width, $L$, of the soliton as a function of $n$ and show that $L \rightarrow \infty$ in the harmonic limit (i.e., when a soliton solution is no longer supported by the equations of motion) and $L \rightarrow 1$ as $n \rightarrow \infty$, i.e., in the limit of hardcore repulsion between the spheres. We also establish that existing long wavelength analysis of the propagation of an impulse in a Hertzian chain leads to solitons and that the results thus obtained are reasonably good in the $n=2.5$ case but become unreliable for $n \gg 3$. (3) The problem of crossing of two solitons that are initiated at the opposite ends of a chain is shown to be distinct from the corresponding problem for solitons in continuum systems. Hertzian solitons lead to the spawning of new secondary solitons at the point of intersection. (4) The decay of the amplitude of a soliton-like object and the lengthening of its tail during propagation through loaded chains is discussed in some detail and in this context we probe the acceleration correlations of individual grains in loaded chains for various conditions of loading and impact. These studies are crucial to the eventual development of a theoretical framework to describe the problem of propagation of solitons and soliton-like excitations in Hertzian chains under various conditions of loading.

The key open question concerning soliton propagation is the construction of an exact solution to Eq. (3) that will describe the properties of the Hertzian solitons and soliton-like objects for any $n$ and for any loading condition. Such a solution may also provide an important step towards developing a theory of non-linear response in these strongly non-equilibrium, interacting, many-particle systems.

\section{Acknowledgements}

This research was supported in part by the U.S. Army (DACA-39-97-K0026) and by the U.S. Department of Energy (Contract No. DE-AC04-94AL8500). Sandia is a multiprogram laboratory operated by Sandia Corporation, a Lockheed Martin Company, for the

\section{References} United States Department of Energy under contract DE-AC04-94AL85000.

[1] G. Eilenberger, Solitons, Springer, Berlin, 1983.

[2] J.S. Russell, Report of the fourteenth meeting of the British Association for the Advancement of Science, York, September 1844, London. Plates XLVII-LVII, pp. 311-390. 
[3] V.F. Nesterenko, J. Appl. Mech. Tech. Phys. (USSR) 24(5) (1983) 733.

[4] A.N. Lazaridi, V.F. Nesterenko, J. Appl. Mech. Tech. Phys. 26 (1985) 405.

[5] V.F. Nesterenko, J. de Physique IV, C8 4 (1994) 729.

[6] R.S. Sinkovits, S. Sen, Phys. Rev. Lett. 74 (1995) 2686.

[7] S. Sen, R.S. Sinkovits, Phys. Rev. E 54 (1996) 6857.

[8] C. Coste, E. Falcon, S. Fauve, Phys. Rev. E 56 (1997) 6104.

[9] R.S. MacKay, Phys. Lett. A 251 (1999) 191.

[10] S. Sen, M. Manciu, Physica A 268 (1999) 644.

[11] L.D. Landau, E.M. Lifshitz, Theory of Elasticity, 2nd Edition, Pergamon, Oxford, 1970.

[12] D.A. Spence, Proc. R. Soc. Lond. A 305 (1968) 55.

[13] J.D. Goddard, Proc. Roy. Soc. (Lond.) A 430 (1990) 105.

[14] S. Sen, M.J. Naughton, Nonlinear Acoustic Detector of Buried Objects, U.S. Patent Application, Pending, 1999.

[15] M.J. Naughton, R. Shelton, S. Sen, M. Manciu, in Detection of Abandoned Landmines, IEE Conf. Pub. No. 458, 249, IEE, London, 1998.

[16] M. Manciu, S. Sen, A.J. Hurd, Physica A 274 (1999) 607.

[17] M.P. Allen, D.J. Tildesley, Computer Simulation of Liquids, Clarendon, Oxford, 1987.

[18] S. Sen, M. Manciu, J.D. Wright, Phys. Rev. E 57 (1998) 2386.

[19] M. Manciu, S. Sen, unpublished.

[20] J.B. Hong, J.-Y. Ji, H. Kim, Phys. Rev. Lett. 82 (1999) 3058.

[21] M.H. Lee, Phys. Rev. Lett. 49 (1982) 1072.

[22] M.H. Lee, Phys. Rev. B 26 (1982) 2547.

[23] M.H. Lee, J. Math. Phys. 24 (1983) 2512.

[24] J. Florencio Jr., M.H. Lee, Phys. Rev. A 31 (1985) 3231.

[25] S. Sen, Physica A 186 (1992) 285.

[26] V.N. Tehan, M.S. Thesis, SUNY, Buffalo, 1999.

[27] M. Manciu, V.N. Tehan, S. Sen, unpublished. 